\title{
Clustering and General Self-Organized Tree- based Energy-Balance Routing Protocol for Wireless Sensor Network (CGSTEB)
}

\author{
Avneet Pannu \\ M-tech Student \\ DAV Institute of Engineering \& Technology. \\ Jalandhar, India.
}

\author{
Dinesh Kumar \\ Associate Professor \\ DAV Institute of Engineering \&Technology. \\ Jalandhar, India.
}

\begin{abstract}
WSNs have become major area of research in computational theory due to its wide range of applications. But due to limited battery power the energy consumption has become major limitations of WSNs protocols. Though many protocols has been proposed so far to improve the energy efficiency further but still much enhancement can be done. Although GSTEB has shown quite significant results over available protocols but it can be further improved using clustering based mechanism. This paper has proposed a new clustering and tree based routing protocol for wireless sensor networks. The proposed technique utilize the leach based clustering protocol and improves the GSTEB further by increasing the stability period. The experimental results have shown the significant improvement.
\end{abstract}

Keywords - Energy-balance, network lifetime, clustering, self organized, tree, wireless sensor network.

\section{INTRODUCTION}

Wireless sensor network (WSN) is a system that is composed of a large number of low-cost micro-sensors nodes. This network is used to collect and send various kinds of messages to a base station [23]. With the advancement in Micro-Electro-Mechanical Systems (MEMS)-based sensor technology, low-power wireless communication and low-power digital electronics; it is now feasible to produce wireless sensor nodes in quantity at low price [11]. Wireless sensor network systems have been widely used for monitoring environmental or physical properties over a large area, e.g., temperature, pressure, luminosity and vibration [18].Sensor nodes are widely used to collect information in many applications such as surveillance [8], tracking at critical facilities [9], volcano monitoring [13], permafrost monitoring [17] and monitoring animal habitats [3]. One of the major challenges that are yet to be sorted out in wireless sensor network is the lack of energy efficiency which retards

or reduces the lifetime of the network. WSN consists of low-cost sensor nodes having limited battery power, and the battery replacement is not simple for WSN having thousands of physically embedded nodes, so an energy efficient routing protocol should be employed to have a long network lifetime.

To achieve the aim, we need not only to reduce total energy consumption but also to balance the load in the network [23].
Usually there are two definitions for network lifetime:

a) The time from the beginning of the network operation to the death of the first node in the network.

b) The time from the beginning of the network operation to the death of the last node in the network [1].

To achieve the longer network lifetime for various applications an energy saving technique should be used. The objective is to maximize the lifetime of the network under a given workload. This is accomplished by distributing the workload as equivalently as possible over the whole network [18].The most widely employed energy saving technique is the data aggregation. It requires the data fusion processing in order to reduce the data redundancy collected from the sensor nodes. Data aggregation helps to reduce the traffic load thereby conserving the energy in the sensor nodes[21].Various network architectures and protocols have thus been developed so far to organize the energy efficiently and operate the sensor network [7-16].To overcome the problem of transmission delay and data loss caused due to node failure in the root to sink, cluster based aggregation method is widely used. In large sized network, efficient communication of data to the sink requires to find the best optimal path according to the number of hops in the network. It reduces the efficiency and increases the communication cost. In such a state instead of communicating data individually and directly to sink, it can be aggregated at cluster head, and transmitting the compressed data to sink [22].Clustering technique is enormously effective in broadcasting and data querying [14]. Cluster-heads will help to broadcast messages to the sink by collecting the data from own clusters. Clustering process works with the regional restrictions to enhance data aggregation efficiency and reduce energy consumption thereby extending the network lifetime [20].

In this paper, we propose a modified approach for General Self-Organized Tree based Energy Balance routing protocol (GSTEB) with clustering mechanism (CGSTEB).

The remainder of the paper is organized as follows: Section II presents the related work. Section III presents the proposed scheme and section IV describes the simulation results. Finally, Section V concludes the paper and outlines the future research work. 


\section{RELATED WORK}

GSTEB (General Self-Organized Tree-Based EnergyBalance Routing Protocol for Wireless Sensor Network)[23] is a dynamic protocol in which routing tree is build using the process where in each round, BS selects a root node and broadcasts this assortment to all sensor nodes in the network. Then, each node selects its own parent by considering itself and its neighbor's information. Simulation results have shown that GSTEB gives a better performance than other protocols in balancing energy consumption, thereby extending the network lifetime. In LEACH (Low-Energy Adaptive clustering Hierarchy)[2] sensors nodes are organized to form clusters and in each cluster one sensor node acts as the cluster-head. In LEACH protocol the first node dies over 8 times later than the first node death in direct transmission and the last node dies 3 times later than the last node death in other protocols. HEED (hybrid, energy-efficient, distributed clustering algorithm)[6] is an improvement over the LEACH protocol on the manner of how the cluster heads are selected. HEED protocol ensures that there is only one $\mathrm{CH}$ within a certain range, so there is uniform distribution of the cluster heads in the network which will result in minimum energy consumption thereby enhancing the network lifetime. HEED protocol is suitable only when the nodes have different initial energy. PEDAP (Power Efficient Data Gathering and Aggregation in Wireless Sensor Networks)[5] is a tree-based routing protocol that makes all the sensor nodes in the network form a minimum spanning tree which costs minimum energy consumption for data transmitting. PEGASIS (Power-Efficient Gathering in Sensor Information Systems) [4] is a near Optimal power efficient chain-based protocol that uses GREEDY algorithm. In PEGASIS protocol all the sensor nodes in the network form a chain in which, the (I mod $\mathrm{N})$ th node is selected as the leader, which directly communicates with the base station in round i. $\mathrm{N}$ is the total amount of nodes. PEGASIS protocol reduces the amount of data for long-distance transmission which prolongs the network lifetime. Tree-Based Clustering (TBC) [19] is an improvement over LEACH protocol. TBC forms various clusters in the same way as LEACH forms. In $\mathrm{TBC}$, the nodes within a cluster construct a routing tree where the cluster-head is the root of the tree. The height and the number of levels of the tree is decided according to the distance of the member nodes to the cluster head.TBC is an excellent protocol in which each node records the information of its neighbor nodes and accordingly builds topography, which is similar to GSTEB. TREEPSI (Treebased Efficient Protocol for Sensor Information)[15], selects a root node before data transmission occurs. There are two methods for building the tree path. One way is computing the path centrally by the sink. The other way is to run the same tree construction algorithm in each sensor node. In the initial phase, the root node using the standard tree traversal algorithm visits other nodes. Then in the data transmission phase, from the leaf nodes forwards the data to the root node which sends the collected data to the sink. The process is repeated until the root node dies. Simulation results has shown that communication distance for transferring data between the nodes in TREEPSI is shorter than PEGASIS, so TREEPSI reduces the power consumption about $30 \%$ as compared to PEGASIS. PEACH, (Proxy-Enable Adaptive Clustering Hierarchy for wireless sensor network)[10] is an improvement over the LEACH protocol. In PEACH proxy node is selected which is assumed as the current cluster head having week power during one communication round. It is based on the consensus of healthy nodes for detecting and manipulating the failure of any cluster-head. PEACH protocol shows an improvement in the network lifetime by reducing the overhead of re-clustering. EDACH, (Energy-Driven Adaptive Clustering Hierarchy) [12] is based on the consensus of healthy nodes for detecting and manipulating faults in any cluster head. This protocol employs simulation-based fault injection method for the performance evaluation. It increases the reliability and lifetime of the network even in the presence of clusterheads faults. EDACH extends the lifetime of LEACH up to about 50\%. DEEC, (distributed energy-efficient clustering algorithm) [14] elects the $\mathrm{CHs}$ by the probability based on the ratio between the residual energy of each node and the network average energy. In DEEC protocol, cluster-heads having high initial and residual energy have more chances of being selected as the cluster-heads. DEEC achieves longer network lifetime than the current clustering protocols.

\section{PROPOSED ALGORITHM}

This section contains the various steps required to successfully accomplish the objectives of this paper.

$\operatorname{Begin} \operatorname{GSTEB}(n, E o)$

1. Deploy sensor nodes randomly:

$$
\begin{aligned}
& \text { For } i=1: n \\
& W(i) \cdot x d=\text { rand; } \\
& W(i) \cdot y d=\text { rand; } \\
& W(i) \cdot E=E o ; \\
& \text { end. }
\end{aligned}
$$

Where $E_{O}$ is the initial energy, $W(i)$ represents the sensor nodes, $x d \& y d$ representing the dimensions in $\mathrm{x}$-axis and $\mathrm{y}$-axis respectively.

\section{ApplyT(n) function to elect $\mathrm{CHs}$.}

The cluster-head is decided in the same way as LEACH. $5 \%$ of the nodes are selected as the cluster-heads. For this, decision is made by node $n$ choosing the random number between 0 and 1 . If the number is less than the present threshold $T(n)$, then the node becomes cluster head for the current round. The threshold of node $n$ is set as follows:

$$
T(n)= \begin{cases}\frac{P}{1-P *\left(r \bmod \frac{1}{p}\right)} & \text { if } n \in G \\ 0 & \text { otherwise }\end{cases}
$$

Where $P$ is the desired percentage of the clusterheads, $r$ represents the current round and $G$ represents the set of nodes that have not been the clusterhead in the last $1 / P$ rounds. This algorithm ensure that all nodes in the network become the cluster-head exactly once within $1 / P$ rounds.

3. Associate nodes with respective $\mathrm{CHS}$

$$
\text { For } i=1: n
$$$$
\text { If } W(i) \text {. Type }=\text { ' } N C H \text { ' }
$$$$
d=\sqrt{\begin{array}{c}
\left(W(i) \cdot x d-W(n+1) \cdot(d)^{2}+(W(i) \cdot y d\right. \\
-W(n+1) \cdot y d)^{2}
\end{array}}
$$ 


$$
\begin{aligned}
& W(i) \cdot M D=d \\
& W(i) \cdot \text { root }=n+1 \\
& \text { For } j=1 \text { : length }(C H s) \\
& d=\sqrt{\left(W(i) \cdot x d-W(n+1) \cdot d^{2}+\right.} \\
& \text { if } d<W(i) \cdot y d-W(C H s(j) \cdot x d \\
& W(i) \cdot M D=d \\
& W(i) \cdot r o o t=j ; \\
& \text { end } \\
& \text { end } \\
& \text { end } \\
& \text { end }
\end{aligned}
$$

Where ' $N C H$ ' represents the non Cluster heads, $d$ is the distance and $M D$ represents the minimum distance and root is the root cluster head.

4. Evaluate and update energy consumption. if $d<=d_{o}$

$W(i) \cdot E=W(i) \cdot E-((T X E+E D A) *(k)+$ ef $s * k *(d * d))$;

else

$W(i) \cdot E=W(i) \cdot E-((T X E+E D A) *(k)+$ $a m p * k *(d * d * d * d))$;

end.

Where TXE represents transmitter energy, $E D A$ represents effective data aggregation, $\mathrm{K}$ is the packet size, efs represents the free space and amp is the multipath.

5. Check dead nodes.

$$
\text { dead }=0 \text {; }
$$

$i=1: n$

if $W(i) . E<0$

dead $=$ dead +1

end

end.

6. Stopping criteria If dead $=n$

display network lifetime

else

move to step 2 end.

7. Return network lifetime.

\section{COMPARATIVE ANALYSIS AND SIMULATION RESULTS}

A MATLAB simulation of CGSTEB is done to evaluate the performance. In this section we evaluate the performance of the proposed CGSTEB scheme and compare it with the GSTEB on the basis of first node dead time, tenth node dead time and when all nodes are dead at different energy levels from 0.01 to 0.10 . We assume that there are 100 sensor nodes distributed randomly in a $100 \times 100$ region.

4.1 When first node dies: Table 1 show the comparison between GSTEB and CGSTEB when the first node dies at different energy levels and figure 1 shows the comparison graph where $\mathrm{x}$-axis represents the initial energy given and y-axis represents the number of rounds. Red color in the graph represents the CGSTEB and blue color represents the GSTEB.

We can find that CGSTEB performs much better than the GSTEB when first node dies as first node dies much later in CGSTEB than in GSTEB. So we can see that in CGSTEB load is balanced more efficiently.

Table 1. Comparison table when first node dies

\begin{tabular}{|c|c|c|}
\hline Energy level & $\begin{array}{c}\text { The round a first } \\
\text { node begins to die } \\
\text { in GSTEB }\end{array}$ & $\begin{array}{l}\text { The round a first } \\
\text { node begins to die } \\
\text { in CGSTEB }\end{array}$ \\
\hline 0.01 & 19 & 36 \\
\hline 0.02 & 38 & 81 \\
\hline 0.03 & 55 & 129 \\
\hline 0.04 & 77 & 168 \\
\hline 0.05 & 94 & 221 \\
\hline 0.06 & 107 & 271 \\
\hline 0.07 & 129 & 308 \\
\hline 0.08 & 143 & 353 \\
\hline 0.09 & 165 & 382 \\
\hline 0.10 & 179 & 438 \\
\hline
\end{tabular}

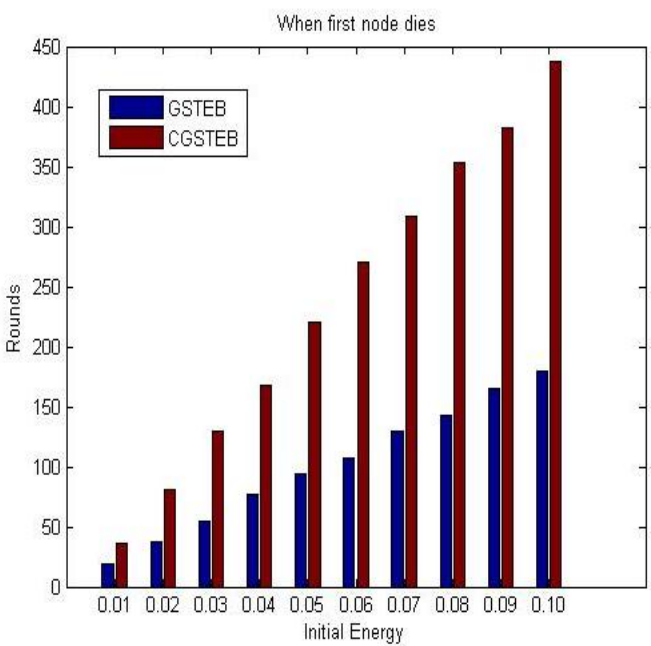

Figure 1.Graph when first node is dead in GSTEB and CGS TEB 
4.2 When tenth node dies: Table 2 show the comparison between GSTEB and CGSTEB when the tenth node dies at different energy levels from 0.01 to 0.10 .

Table 2.Comparison table when tenth node dies

\begin{tabular}{|c|c|c|}
\hline Energy level & $\begin{array}{c}\text { The round tenth } \\
\text { node begins to die } \\
\text { GSTEB }\end{array}$ & $\begin{array}{l}\text { The round tenth } \\
\text { node begins to } \\
\text { die in CGSTEB }\end{array}$ \\
\hline 0.01 & 21 & 40 \\
\hline 0.02 & 44 & 89 \\
\hline 0.03 & 62 & 136 \\
\hline 0.04 & 80 & 183 \\
\hline 0.05 & 105 & 230 \\
\hline 0.06 & 119 & 282 \\
\hline 0.07 & 141 & 325 \\
\hline 0.08 & 160 & 373 \\
\hline 0.09 & 186 & 419 \\
\hline 0.10 & 224 & 476 \\
\hline
\end{tabular}

We can find that is CGSTEB stability period is increased means first node dead time and tenth node dead time is increased significantly thereby balancing the load in wireless sensor network. We can find that in CGSTEB tenth node dies much later than in GSTEB and figure 2 shows the comparison graph when tenth node is dead at different energy levels from 0.01 to 0.10 . In the graph $x$ axis represents the initial energy given and $y$-axis represents the number of rounds. Red color in the graph represents the CGSTEB and blue color represents the GSTEB.

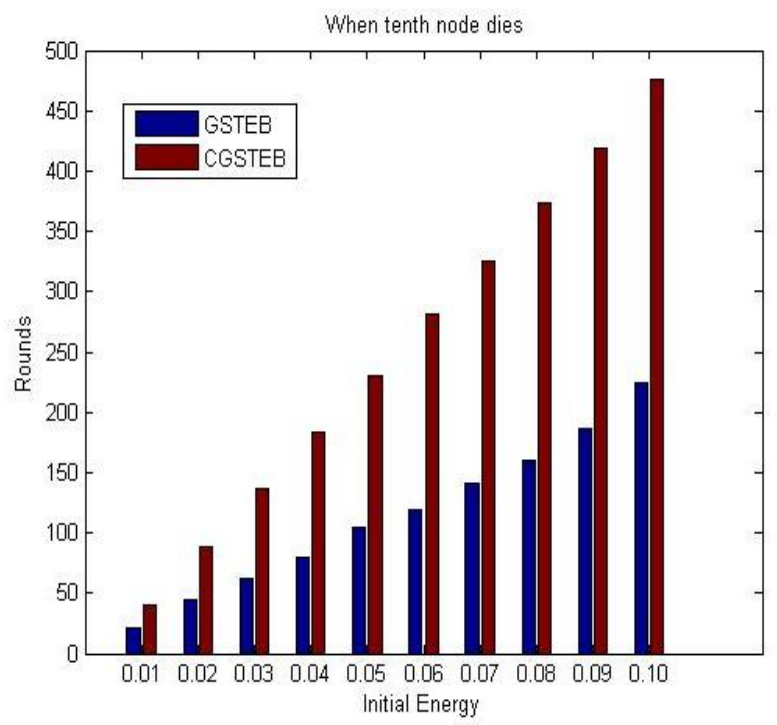

Figure 2.Graph when tenth node dies in GSTEB and CGS TEB

4.3 When all the nodes are dead: Table 3 shows the comparison between GSTEB and CGSTEB when all the nodes are dead at different energy levels from 0.01 to 0.10 .
Table 3.Comparison table when al thel nodes are dead

\begin{tabular}{|c|c|c|}
\hline Energy level & $\begin{array}{l}\text { The round all the } \\
\text { nodes are dead in } \\
\text { GSTEB }\end{array}$ & $\begin{array}{l}\text { The round all the } \\
\text { nodes are dead } \\
\text { in CGSTEB }\end{array}$ \\
\hline 0.01 & 50 & 50 \\
\hline 0.02 & 100 & 100 \\
\hline 0.03 & 150 & 150 \\
\hline 0.04 & 200 & 207 \\
\hline 0.05 & 250 & 254 \\
\hline 0.06 & 300 & 292 \\
\hline 0.07 & 350 & 335 \\
\hline 0.08 & 400 & 392 \\
\hline 0.09 & 450 & 443 \\
\hline 0.10 & 500 & 499 \\
\hline
\end{tabular}

The overall results show that there exist an improvement in stability period of CGSTEB protocol and as the first node and tenth node dies much latter in CGSTEB than in GSTEB so load balancing is more proper in CGSTEB than in GSTEB but in some cases the network lifetime is low in case of clustering based than standard GSTEB, therefore in near future compressive sensing and swarm intelligence technique to find the shortest path will be used to enhance the network lifetime further. Figure 3 shows the comparison graph when all the nodes are dead and $\mathrm{x}$-axis represents the initial energy given and $y$-axis represents the number of rounds. Red color in the graph represents the CGSTEB and blue color represents the GSTEB.

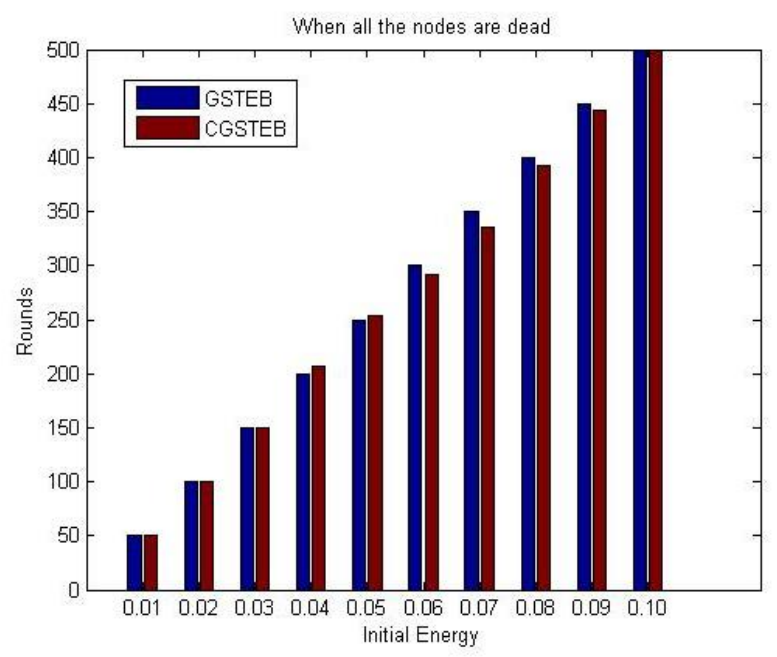

Figure 3.Graph when all nodes are dead in GS TEB and CGS TEB

\section{CONCLUSION AND FUTURE WORK}

In this paper we have proposed a new clustering and tree based routing protocol for wireless sensor networks. Even though GSTEB has shown quite significant results over existing protocols, it has been further enhanced using 
clustering based mechanism. The proposed CGSTEB utilizes the leach based clustering protocol and improved the GSTEB further. Simulation results has shown that the proposed scheme successfully balances the energy consumption among the nodes and there is an improvement in the stability period. The work has not considered the use of compressive sensing and any swarm intelligence technique to find the shortest path between the cluster heads and base station. So in near future we will propose compressive sensing and swarm intelligence based CGSTEB protocol to improve the results further.

\section{REFERENCES}

[1] J. H. Chang and L. Tassiulas, "Energy conserving routing in wireless ad hoc networks," in Proc. IEEE INFOCOM, vol. 1, pp. 22-31, 2000.

[2] W. R. Heinzelman et al, "Energy efficient communication protocols for wireless microsensor networks," in Proc. 33rd Hawaii Int. Conf. SystemSci.,pp.3005-3014,Jan.2000.

[3] A. Mainwaring, J. Polastre, R. Szewczyk, D. Culler, and J. Anderson, "Wireless Sensor Networks for Habitat Monitoring," WSNA'02, Atlanta, Georgia, September 2002.

[4] S. Lindsey and C. Raghavendra, "Pegasis: Powerefficient gathering in sensor information systems," in Proc. IEEE Aerospace Conf., vol. 3, pp. 1125-1130 , 2002.

[5] H. O. Tan and I. Korpeoglu, "Power efficient data gathering and aggregation in wireless sensor networks," SIGMOD Rec., vol. 32, no. 4, pp. 66-71, 2003.

[6] O. Younis and S. Fahmy, "HEED: A hybrid, energy efficient, distributed clustering approach for ad hoc sensor networks," IEEE Trans. Mobile Computing, vol. 3, no. 4, pp. 660-669, 2004.

[7] Q. Jiang and D. Manivannan, "Routing Protocols for Sensor Networks", IEEE Consumer Communications and Networking Conference, pp. 93-98, Sept 2004.

[8] D. Culler, D. Estrin, and M. Srivastava, "Overview of Sensor Networks," IEEE Computer, August 2004.

[9] N. Xu, S. Rangwala, K. Chintalapudi, D. Ganesan, A. Broad, R. Govindan, and D. Estrin, "A Wireless Sensor Network for Structural Monitoring," in Proceedings of the ACM Conference on Embedded Networked Sensor Systems, Baltimore, MD, November 2004.

[10] K. T. Kim and H. Y. Youn, "PEACH:Proxy-Enable Adaptive Clustering Hierarchy for Wireless Sensor network," Proceeding of the 2005 International Conference On Wireless Network, pp. 52-57, , June 2005.
[11] K. Akkaya and M. Younis, "A survey of routing protocols in wireless sensor networks," Elsevier Ad Hoc Network J., vol. 3/3, pp. 325-349, 2005.

[12] K. T. Kim and H. Y. Youn, "Energy-Driven Adaptive Clustering Hierarchy (EDACH) for Wireless Sensor Networks", LNCS, vol. 3823, pp. 1098-1107, Dec 2005.

[13] G. Werner-Allen, K. Lorincz, J. Johnson, J. Lees, and $\mathrm{M}$. Welsh. Fidelity and yield in a volcano monitoring sensor network. In OSDI'06, Berkeley, CA, USA, USENIX Association, 2006.

[14] L. Qing et al, "Design of a distributed energy efficient clustering algorithm for heterogeneous wireless sensor networks," ELSEVIER, Computer Communications, pp. 2230-2237, 2006.

[15] S. S. Satapathy and N. Sarma, "TREEPSI: tree based energy efficient protocol for sensor information", Wireless and Optical Communications Networks 2006 IFIP International Conference, April 2006.

[16] E. Hansen, M. Nolin, M. Bjorkman, "A Study of Maximum Lifetime Routing in Sparse Sensor Networks", in Proceedings of International Conference on Complex, Intelligent and Software Intensive Systems (CISIC), Spain, pp. 449-454, Mar 2008.

[17] J. Beutel, S. Gruber, A. Hasler, R. Lim, A. Meier, C. Plessl, I. Talzi, L. Thiele, C. Tschudin, M. Woehrle, and M. Yuecel. PermaDAQ: A scientifi instrument for precision sensing and data recovery in environmental extremes. In IPSN'09, San Francisco, CA, USA, 2009.

[18] D. Hasenfratz et al, "Analysis, Comparison, and Optimization of Routing Protocols for Energy Harvesting Wireless Sensor Networks,"IEEE,Int.Conf on Sensor Networks,Ubiquitious and Trustworthy Computing, pp.19-26,2010.

[19] K. T. Kim and H. Y. Youn, "Tree-Based Clustering(TBC) for energy efficient wireless sensor networks," in Proc. AINA 2010, pp. 680-685, 2010.

[20] J. Xibei et al, "Research of Data Aggregation Routing Protocol in WSN Data-Related Applications,"IEEE,pp. 467-451,2010

[21] B.S. Mathapati et al, "Energy Efficient Reliable Data Aggregation Technique for Wireless Sensor Networks," Int. Conf on Computing Sciences, pp. 153158,2012 .

[22] D. Mantri et al, "Grouping of Clusters for Efficient Data Aggregation (GCEDA) in Wireless Sensor Network," IEEE, pp. 132-137, 2013.

[23] Z. Han et al, “A General Self-Organized Tree-Based Energy-Balance Routing Protocol for Wireless Sensor Network," IEEE Transactions on Nuclear Science, VOL. 61, pp. 732-740, April 2014. 